\title{
Transplantation of placenta-derived mesenchymal stem cells reduces hypoxic-ischemic brain damage in rats by ameliorating the inflammatory response
}

\author{
Hongfang Ding ${ }^{1}$, Hui Zhang ${ }^{2}$, Huifang Ding ${ }^{1}$, Dong $\mathrm{Li}^{3}$, Xinhao Yi ${ }^{1}$, Xiaoxu Ma ${ }^{1}$, Ruijuan Li $^{1}$, \\ Mei Huang ${ }^{1}$ and Xiuli $\mathrm{Ju}^{3}$
}

Hypoxic-ischemic brain damage (HIBD) is a common cause of infant death. The purpose of our research was to explore the immunoregulatory mechanism of placenta-derived mesenchymal stem cells (PD-MSCs) in HIBD treatment.

Seven-day-old rat pups were randomly divided into HIBD, PD-MSC, fibroblast, and control groups. Forty-eight hours after HIBD induction, cells at a density of $5 \times 104$ cells/10 $\mu \mathrm{l}$ were injected into the cerebral tissue in the PD-MSC and fibroblast groups. The TNF- $\alpha$, interleukin- 17 (IL-17), interferon- $\gamma$ (IFN- $\gamma$ ), and IL-10 levels were detected through quantitative real-time polymerase chain reaction (RT-PCR) and enzyme-linked immunosorbent assay (ELISA).

Regulatory T cell (Tregs) populations were detected through flow cytometry, and forkhead box P3 (Foxp3) was measured through western blot analysis. Behavioral tests and gross and pathological examinations showed that PD-MSC treatment exerted significantly stronger neuroprotective effects than the other treatments. The expression levels of pro-inflammatory cytokines were substantially upregulated after $\mathrm{HI}$ injury. Compared with fibroblast treatment, PD-MSC treatment inhibited the production of pro-inflammatory cytokines and increased the production of IL-10 in the ischemic hemispheres and peripheral blood serum (all $P<0.01$ ). Flow cytometry results showed a notable increase in the number of Tregs within the spleen of the HIBD group. Moreover, the number of Tregs and the Foxp3 expression levels were higher in the PD-MSC treatment group than in the HIBD and fibroblast groups (all $P<0.01$ ). Our research suggests that the mechanism of PD-MSC treatment for HIBD partially involves inflammatory response suppression.

Cellular \& Molecular Immunology (2017) 14, 693-701; doi:10.1038/cmi.2015.99; published online 28 December 2015

Keywords: cytokine; hypoxia-ischemia; mesenchymal stem cell; neonatal rat; regulatory T cell

\section{INTRODUCTION}

Hypoxic-ischemic brain damage (HIBD) is a type of newborn brain damage that is caused by perinatal asphyxia and hypoxic ischemic during the perinatal period. Approximately $15-20 \%$ of HI-injured infants die, and at least $25 \%$ of survivors have long-term neurological disorders, such as cerebral palsy, epilepsy and mental retardation. ${ }^{1}$ However, the exact pathogenesis of HIBD has not been thoroughly ascertained. At present, treatment options for HI injury are largely supportive. ${ }^{2}$ The economic and emotional costs of caring for children affected by HIBD are enormous, and seeking therapeutic strategies to ameliorate the severity of HI-related brain injury remains of important significance.
The inflammatory response to HIBD has recently attracted considerable attention. In the acute phase of cerebral infarction, macrophages infiltrate and promote rapid inflammatory responses; however, in the late phases of cerebral infarction, a variety of inflammatory cells, including lymphocytes, and cytokines aggravate ischemic injury. ${ }^{3} \mathrm{~T}$ cells produce various cytokines that can strongly regulate the post-ischemic inflammation. $\mathrm{CD}^{+}$helper $\mathrm{T}$ cells produce interferon- $\gamma(\mathrm{IFN}-\gamma)$, which directly leads to neuron death. $\gamma \delta \mathrm{T}$ cells are the main source of interleukin 17 (IL-17) after ischemic damage. ${ }^{4}$ These cytokines might amplify inflammatory cascades and recruit monocytes and neutrophils to injury sites. However, limited data are available regarding the imbalance between

\footnotetext{
${ }^{1}$ Department of Pediatrics, Shengli Oil field Central Hospital, 31 Jinan Road, Dongying, Shandong, China; ${ }^{2}$ Department of Neurology, Qingdao Municipal Hospital, the Affiliated Hospital of Medical College of Qingdao University, 1 Jiaozhou Road, Qingdao, Shandong, China and ${ }^{3}$ Department of Pediatrics, Qilu Hospital, Shandong University, 107 West Wenhua Road, Jinan, Shandong, China

Correspondence: Prof. X Ju, Department of Pediatrics, Qilu Hospital, Shandong University, 107 West Wenhua Road, Jinan, Shandong 250012, China. E-mail: qlyyjx|@163.com

Received: 29 May 2015; Revised: 29 October 2015; Accepted: 30 October 2015
} 
pro-inflammatory and anti-inflammatory cytokines in the peripheral and central nervous systems after HI injury. Immunoregulatory mechanisms may limit the inflammatory phase and mediate immunosuppression. Regulatory $\mathrm{T}$ cells (Tregs), a T cell subset, play a pivotal role in the pathophysiological process of ischemic stroke. Within the population of $\mathrm{CD} 4{ }^{+} \mathrm{T}$ cells, Tregs are identified by cell-surface expression markers, such as CD25. The transcription factor forkhead box P3 (Foxp3), which is a specific intracellular marker of CD4 ${ }^{+}$ $\mathrm{CD} 25^{+}$Tregs, is the key regulatory gene for the development and function of Tregs. ${ }^{5}$ A recent report has indicated that Tregs provide neuroprotection after brief focal cerebral ischemia. Tregs produce IL-10, which exerts an anti-inflammatory effect by reducing the generation of TNF- $\alpha$ and IFN- $\gamma$. The depletion of the $\mathrm{CD} 25^{+}$population with anti-CD25 mAb significantly increases brain infarct volume and worsens functional outcomes. ${ }^{6}$ In the systemic and central nervous systems, Tregs secrete anti-inflammatory molecules, including IL-10 and transforming growth factor- $\beta$. IL-10, an important mediator of Tregs, has been shown to exert protective effects in central ischemia. ${ }^{7}$ Despite these recent findings, little is known about the distribution, induction, and activation of Tregs after cerebral ischemia. Therefore, the current study sought to analyze the cytokines and activation levels of Tregs in the peripheral lymphatic organs after HI-related brain injury.

Stem cell therapy is effective in restoring tissue and organ injuries in animal models. ${ }^{8}$ Stem cell transplantion plays a neuroprotective and neuroregenerative role in injury central tissues, and is associated with a low risk of rejection and side effects. ${ }^{9,10}$ Bone marrow (BM) is the most common source of mesenchymal stem cells (MSCs). However, the proliferation and differentiation potentials of BM-MSCs are also weak. The invasive procedures and increased probability of viral infection limit the further clinical application of BM-MSCs. ${ }^{11}$ Several laboratories have successfully isolated multi-potent differentiatied cells from discarded tissues after delivery, such as the umbilical cord, cord blood, and placenta. These cells are capable of self-renewal, high proliferation, multilineage differentiation, and immunoregulation. These properties make placenta derived-(PD) MSCs a prospective alternative source of MSCs for fundamental research and clinical applications. ${ }^{12}$ The mechanism of stem cell transplantation in the treatment of central injury involves alternative nerve cells, trophic factor secretion, and local microcirculatory improvements. ${ }^{13}$ To date, it is considered a multifaceted joint comprehensive repair mechanism. Whether PD-MSC treatment for HIBD is partially mediated by suppressing immune responses warrants further investigation. Therefore, our research explored the potential therapeutic efficacy and possible mechanism of PD-MSC treatment in ameliorating autoimmune progression in HIBD rats.

\section{MATERIALS AND METHODS}

\section{Animal care}

Animals from the experimental animal center of Shandong University were maintained in the Shandong University Qilu
Hospital animal facility. All experimental protocols were approved by the Shandong University Institutional Animal Care and Use Committee.

\section{Cell culture}

Adherent cells derived from the placenta were cultured in accordance with our previously published protocol. ${ }^{14}$ Priming was conducted by culturing with $10 \%$ fetal bovine serum (Gibco Invitrogen, Carlsbad, CA, USA), $100 \mathrm{U} / \mathrm{ml}$ penicillin, and $100 \mu \mathrm{g} / \mathrm{ml}$ streptomycin. To track grafted MSCs, cells at the third passage were transfected with a lentivirus carrying the green fluorescent protein (GFP) gene. The results of the in vitro GFP gene transfection were observed with fluorescence microscopy.

\section{HIBD animal model and transplantation}

A rat model of HIBD was established as previously described. ${ }^{14,15} \mathrm{P} 7$ Wistar rat pups were anesthetized, and the left common carotid arteries were cut. After the surgical procedure, the young rats were placed in a jar full of warmed, humidified $8 \%$ oxygen (balanced with nitrogen) at $1.5 \mathrm{l} / \mathrm{min}$ for $2.5 \mathrm{~h}$. After HIBD was induced, the newborn animals were randomly divided into three groups $(n=20)$ : HIBD, HIBD + PD-MSC, and HIBD + fibroblast groups. For the PD-MSC group, the skull bregma ( $3 \mathrm{~mm}$ from the front of the herringbone stitch, $2 \mathrm{~mm}$ from the left of the midline, depth of $2 \mathrm{~mm}$ ) was determined, and a single cell suspension $\left(5 \times 10^{4}\right.$ cells $/ 10$ $\mu \mathrm{l}$ ) of the third passage PD-MSCs was transplanted at $48 \mathrm{~h}$ after HIBD with a stereotaxic apparatus. The injection rate was $1 \mu \mathrm{l} /$ min with a total volume of $2 \mu \mathrm{L}$. The fibroblast group received the same volume of fibroblasts at the same time.

\section{Neurobehavioral tests}

Behavioral tests, including the hanging wire and vertical pole tests, were performed for all of the rat pups from 3 to $21 \mathrm{~d}$ after HI injury. ${ }^{16}$

The hanging wire test evaluates neuromuscular development. First, we took a wire cage lid and put the experimental animal on it. Then, we gently shook it three times and turned it upside down. Rats supported themselves with their limbs to prevent themselves from falling. We placed a box filled with sawdust at the base to protect the falling rats. The distance from the lid was $20 \mathrm{~cm}$. A stopwatch was used to quantify the time until the experimental animal dropped from the lid.

In the vertical pole test, we tested the movement balance of the experimental animals. First, we placed an experimental animal on the center of a pole. Then, we gently moved the pole to a vertical position from the horizontal position. We recorded the angle at which the experimental animal dropped off the pole.

\section{Histological examination}

Five rats that were randomly selected from each group were anesthetized with $4 \%$ paraformaldehyde after the behavioral tests $21 \mathrm{~d}$ after HI injury. The brains were collected, fixed, 
and paraffin-embedded. Coronal brain sections with a thickness of $5 \mu \mathrm{m}$ were prepared. Each specimen was randomly selected, and three slices were dewaxed, hydrated, and counterstained with hematoxylin and eosin staining. The number of GFP-positive cells was counted in the same area of three coronal brain sections and observed with microscopy.

\section{The cytokine mRNA expression levels}

The inflammatory cytokine expression levels in the brain tissues were detected through quantitative real-time polymerase chain reaction (RT-PCR) in the HIBD group at different time points ( $3 \mathrm{~h}, 6 \mathrm{~h}, 24 \mathrm{~h}, 3 \mathrm{~d}$, and $5 \mathrm{~d}$ after $\mathrm{HI}$ ). We also assessed the cytokine mRNA expression levels at $3 \mathrm{~d}$ after transplantation in the four groups. In brief, total RNA was extracted from tissue dissected from the ipsilateral hemisphere in TRIzol (Invitrogen). Total RNA (5000 ng) was used to synthesize first-strand cDNA by SuperScript polymerase through the First-Strand Synthesis System for RT-PCR (Thermo Fisher Scientific, Inc., Waltham, MA, USA). The cDNA product was amplified through PCR. The mRNA expression levels were quantified using a SYBR Green two-step kit. A PCR mixture was prepared with SYBR Green PCR Master Mix (Thermo Fisher Scientific, Inc., Waltham, MA, USA). Primers used were shown in Table 1. After an initial denaturation at $95^{\circ} \mathrm{C}$ for $60 \mathrm{~s}$, 40 cycles of a two-cycle procedure (denaturation at $95^{\circ} \mathrm{C}$ for 15 $\mathrm{s}$, annealing and extension at $60^{\circ} \mathrm{C}$ for $1 \mathrm{~min}$ ) were performed. The expression of each gene relative to $\beta$-actin was determined through the 2-dCT method. The data were analyzed using Sequence Detection Software 1.4 (Applied Biosystems). The mRNA expression levels were determined as fold differences compared with the control group.

\section{Analysis of cell populations through fluorescence-activated cell sorting analysis}

Splenic single-cell suspensions were obtained on $7 \mathrm{~d}$ after HI injury through mechanical disruption for flow cytometry. Cells were washed with phosphate-buffered saline (PBS) and then stained with a combination of monoclonal antibodies specific to CD4 and CD25 (BD, Franklin Lakes, NJ, USA). After incubation with monoclonal antibodies, the cells were analyzed on a flow cytometer (Cytometer 1.0, CytomicsTM FC500, Beckman Coulter). Forward and side scatter parameters were chosen to

Table 1 Primers used for RT-PCR analysis

\begin{tabular}{|c|c|c|}
\hline Gene & Primer & Sequence \\
\hline IL-17 & $\begin{array}{l}\text { Sense primer } \\
\text { anti-sense primer }\end{array}$ & $\begin{array}{l}\text { 5'-TGAACCTGGAGGCTACAGTGAA-3' } \\
\text { 5'-GGCCTCGGCGTTTGG-3' }\end{array}$ \\
\hline IFN- $\gamma$ & $\begin{array}{l}\text { Sense primer } \\
\text { anti-sense primer }\end{array}$ & $\begin{array}{l}\text { 5'-TACACGCCGCGTCTTGGT-3' } \\
\text { 5'-GAGTGTGCCTTGGCAGTAACAG-3' }\end{array}$ \\
\hline TNF- $\alpha$ & $\begin{array}{l}\text { Sense primer } \\
\text { anti-sense primer }\end{array}$ & $\begin{array}{l}\text { 5'-GGGTGATTGGTCCCAACAAG-3' } \\
\text { 5'-GGGTCTGGGCCATGGAA-3' }\end{array}$ \\
\hline IL-10 & $\begin{array}{l}\text { Sense primer } \\
\text { anti-sense primer }\end{array}$ & $\begin{array}{l}\text { 5'-CCCTGGGAGAGAAGCTGAAGA-3' } \\
\text { 5'-CACTGCCTTGCTTTTATTCTCACA-3' }\end{array}$ \\
\hline$\beta$-actin & $\begin{array}{l}\text { Sense primer } \\
\text { anti-sense primer }\end{array}$ & $\begin{array}{l}\text { 5'-TCTGTGTGGATT GGTGGCTCTA-3' } \\
\text { 5'-CTGCTTGCTGATCCACATCTG-3' }\end{array}$ \\
\hline
\end{tabular}

identify lymphocytes. The data were analyzed with the CellQuest software.

\section{Inflammatory cytokine level measurements}

We assessed the cytokine expression levels on $5 \mathrm{~d}$ after HI. The serum was collected and immediately frozen until the protein concentration quantification was performed using kits for TNF- $\alpha$, IFN- $\gamma$, IL-10 (R\&D Systems, USA), and IL-17 (eBioscience, San Diego, California, USA). These procedures were performed according to the manufacturer's instructions.

\section{Western blot analysis}

On $7 \mathrm{~d}$ after HI injury, we washed spleen samples using ice-cold PBS. The homogenates were lysed with radioimmunoprecipitation assay buffer (Sigma-Aldrich, St. Louis, MO, USA). All of the samples were assayed for protein concentration using a bicinchoninic acid assay (Pierce Chemical Co., Rockford, IL, USA) and then stored at $-80^{\circ} \mathrm{C}$. We separated proteins through SDS-polyacrylamide gel electrophoresis. Then, we transferred samples to a polyvinylidene difluoride membrane, and we blocked the membrane using $5 \%$ bovine serum albu$\mathrm{min}$ in a PBS/Tween 20 solution. The blots were incubated with antibodies specific to Foxp3 and $\beta$-actin (Sigma-Aldrich) at $4{ }^{\circ} \mathrm{C}$. After washing, the blots were incubated with horseradish peroxidase-conjugated secondary antibodies for $1 \mathrm{~h}$. The bands were visualized through enhanced chemiluminescence (Amersham Biosciences, Pittsburgh, PA, USA) and then exposed to Biomax L film (Kodak, Rochester, NY, USA). The relative densities of the bands were analyzed using the Kodak Digital Science Imaging System.

\section{Statistical analysis}

Statistical analysis was performed using SPSS software version 14.0 (SPSS Inc., Chicago, IL). PCR results were expressed as the mean \pm SE. Dependent variables (levels of various cytokines and Tregs) were presented as the mean \pm SD and analyzed using one-way analyses of variance (ANOVAs) with Tukey's honest significant difference multiple comparisons as the between-subjects factor. Statistical significance was considered as $P<0.05$.

\section{RESULTS}

Characteristics, survival, and migration of PD-MSCs in the HIBD model

MSCs were isolated and cultured from the placentas of pregnant rats. Forty-eight hours after culture, adherent fibroblast-like cells were detected. Cell morphology was uniform, spindle-shaped, and partly spiral-like after 2 weeks (Figure 1a and b). The flow cytometry results showed the positive CD29 $(97.16 \pm 4.61 \%)$, CD44 (98.69 $\pm 4.10 \%)$, CD90 (96.36 \pm $4.53 \%)$, and CD105 (95.75 $\pm 5.52 \%)$ expression levels and the negligible CD45 expression levels $(0.75 \pm 0.28 \%$; Figure 1c). Under the appropriate induction conditions, the PD-MSCs differentiated into osteoblasts, chondrocytes, and adipocytes (Figure 1d). These results were in accordance with the international MSC standards. The GFP transfection 

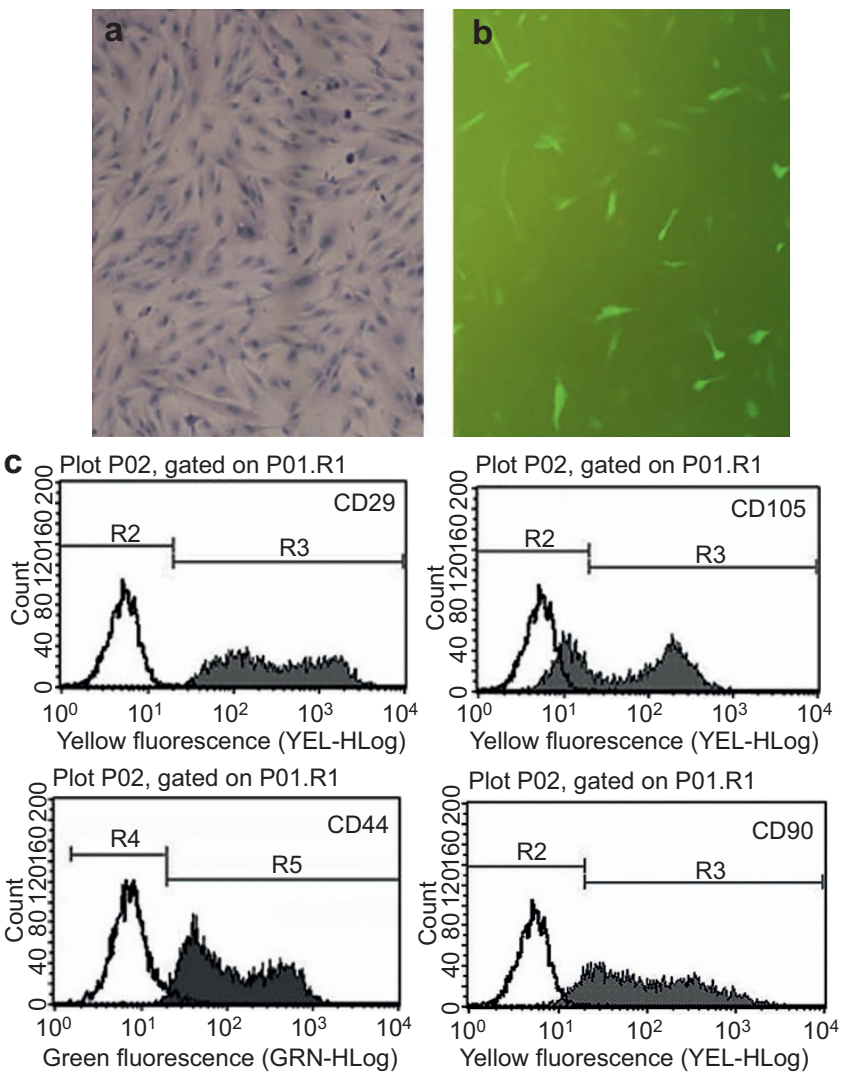

d

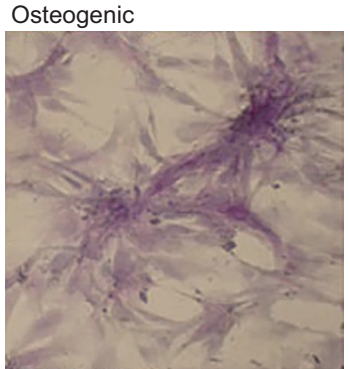

\section{Adipocytic}

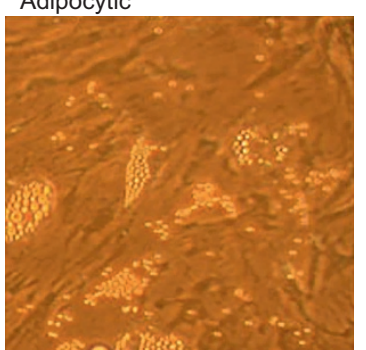

Chondrogenic

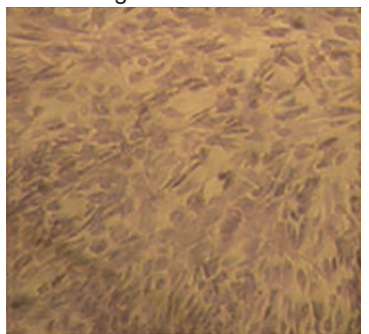

Merged
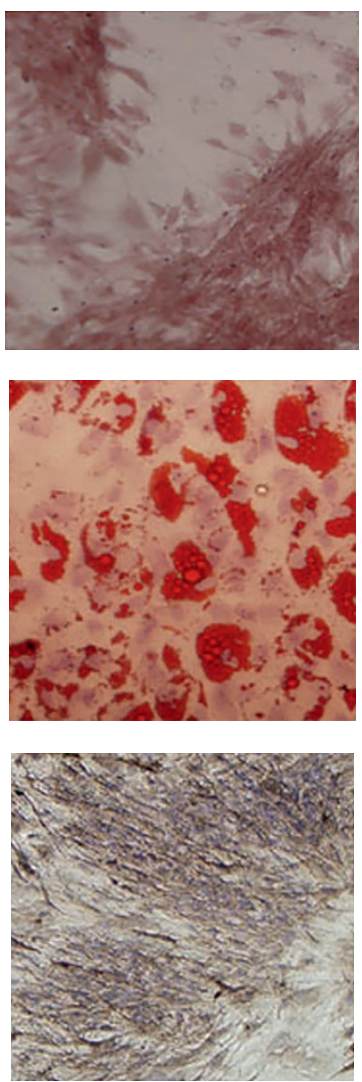

e

GFP

DAPI
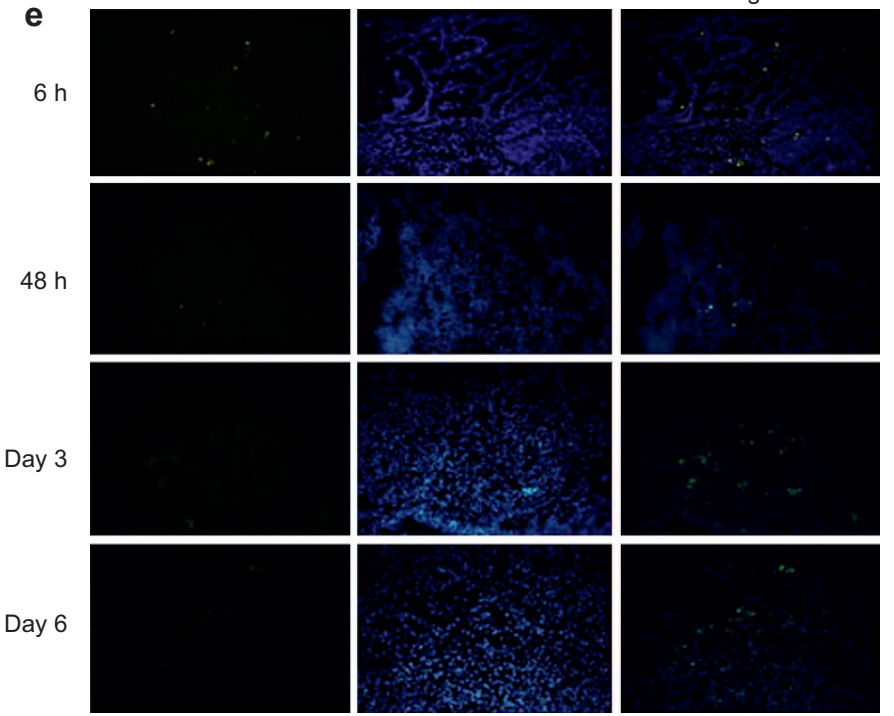

Figure 1 Characteristics, survival, and migration of PD-MSCs in the HIBD model. (a and b) Cell morphology was uniform spindle-shaped and partly spiral-like after 2 weeks; (c) Flow cytometry showed the positive CD29, CD44, CD90, and CD105 expression levels and the negligible CD45 expression; (d) Differentiation into osteoblasts, chondrocytes, and adipocytes (original magnification: $\times 200$ ); (e) GFP-positive PD-MSCs were found in the infarct area. At $6 \mathrm{~h}$ after transplantation, GFP-positive PD-MSCs were primarily found around the injection site. At $6 \mathrm{~d}$ after MSC implantation, the green fluorescent signal was significantly attenuated. The scale bar represents $200 \mu \mathrm{m}$.

efficiency was $92 \%$, which was confirmed by fluorescent microscope observation.

The grafted PD-MSC survival levels were analyzed by detecting GFP to identify the grafted cells. The PD-MSCs survived and migrated toward the damaged tissue. In the HI pups, trans- planted PD-MSCs were found in the needle track and the injection site. Then, the grafted cells were transferred from the injection site to the cortical and periventricular regions. GFPpositive PD-MSCs were found in the infarct area. At $6 \mathrm{~h}$ after transplantation, GFP-positive PD-MSCs were primarily found 
around the injection site. At $6 \mathrm{~d}$ after MSC implantation, the green fluorescent signal was significantly attenuated. GFP-positive cells were observed in the ipsilateral infarct cortex, whereas no staining was observed in the contralateral cortex. These data suggest that grafted PD-MSCs can survive in the postinjury milieu of the injured neonatal cerebral HI (Figure 1e).

\section{Motor function assessment in the HIBD model}

At 3, 9, 15, and $21 \mathrm{~d}$ after $\mathrm{HI}$ injury, the motor skills of animals were evaluated through neurobehavioral, hanging wire, and vertical pole tests. The PD-MSC group had the best recovery from HIBD injury among the three treatment groups.

Hanging wire test: The mean latency time of the HIBD group was distinctly shorter compared with that in the control. The latency in the PD-MSC group was much more prominent than that in the HIBD group (Figure 2a).

Vertical pole test: The motor activities in the HIBD and fibroblast groups clearly decreased compared with the control. Meanwhile, the motor activity was clearly improved in the PDMSC group (Figure 2b).

\section{General observations and histopathologic results}

Newborn rats appeared irritable and cyanotic at the beginning of hypoxia induction, and they gradually experienced suppres-
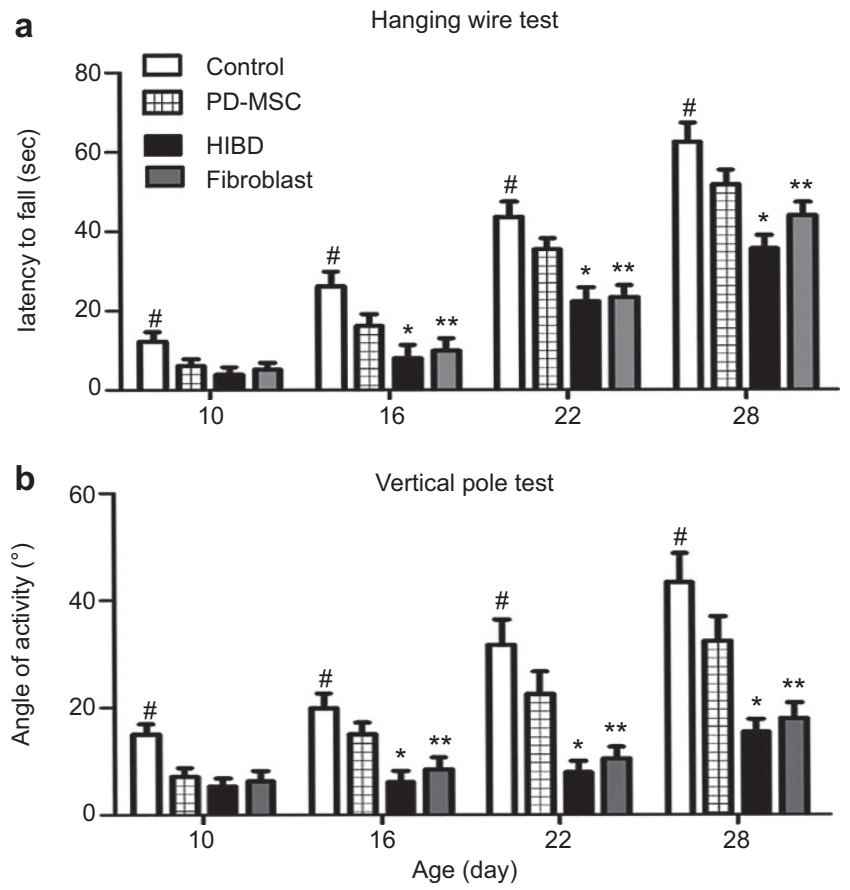

Figure 2 Motor function assessment in the HIBD model. (a) Hanging wire test. Significant time reduction was observed in the HIBD group compared with the control group. The PD-MSC treatment significantly improved the duration of time at which the rats stayed on the lid. (b) Vertical pole test. PD-MSC treatment also significantly improved the duration on pole of the rats after $\mathrm{HI}$ injury. $\#$ : $P<0.01$ vs. the control group; *: $P<0.01$ vs. the HIBD group; **: $P<0.01$ vs. the fibroblast group. sion and even convulsions. The gross morphology of the brains from the HIBD group showed significant atrophy in the left cerebral hemisphere. In addition, the brain morphology in the PD-MSC group was similar to that in the control group brains, without evident cerebral atrophy and edema. Microscopic examination with hematoxylin-eosin staining revealed shrunken neurons and eosinophilic cell bodies in the HIBD group (Figure 3a). After HI induction, the weight gain in the HIBD and HIBD + fibroblast group rats was slower compared with the control group (the control group: P7: $14.00 \pm 2.59 \mathrm{~g}$; P14: $26.55 \pm 4.02$ g; P21: $41.44 \pm 5.90$ g; P28: $57.16 \pm 3.77 \mathrm{~g}$; the HIBD group: P7: $13.85 \pm 1.87 \mathrm{~g}$; P14: $21.18 \pm 4.07 \mathrm{~g}$; P21: 37.23 $\pm 6.34 \mathrm{~g}$; P28: $52.96 \pm 7.67 \mathrm{~g}$; the HIBD+fibroblast group: P7: $13.92 \pm 2.56$ g; P14: $22.2 \pm 2.91$ g; P21: $39.19 \pm 5.99$ g; P28: $52.44 \pm 7.08 \mathrm{~g})$. The body weight of the rats significantly improved in the HIBD+PD-MSC group (P7: $13.87 \pm 2.98 \mathrm{~g}$; P14: $24.24 \pm 2.5$ g; P21: $46.44 \pm 6.46$ g; P28: $61.11 \pm 7.16$ g; Figure $3 b)$.

\section{PD-MSC treatment attenuates systemic inflammation after $\mathrm{HI}$ injury at the gene and protein levels}

The mRNA expression levels of cytokines in the ischemic hemispheres were examined at different time points. The TNF- $\alpha$,
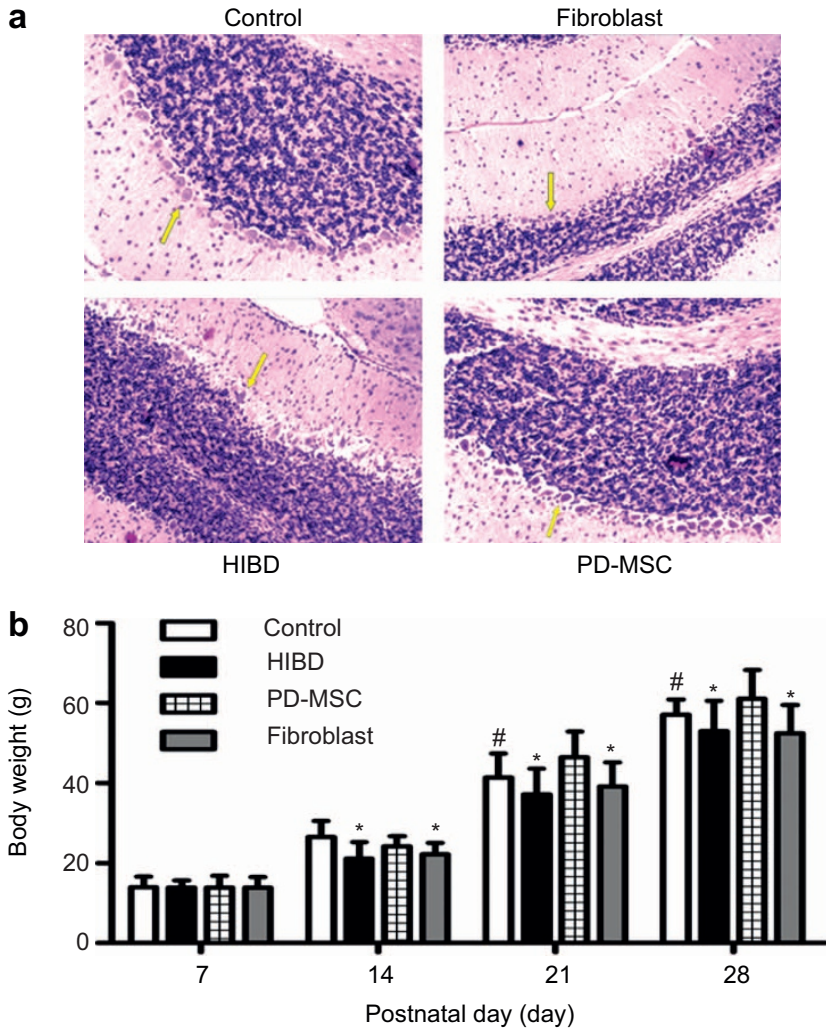

Figure 3 Histopathologic and body weight results in the four groups after cell treatment. (a) Hematoxylin-eosin staining of the Purkinje cells; (b) The weight gain of the rats in the HIBD and fibroblast groups was slower than that of the rats in the control group. The body weight of the rats significantly improved in the PD-MSC group. \#: $P<0.05$ vs. the control group; *: $P<0.01$ vs. the HIBD and fibroblast groups. 
INF- $\gamma$, and IL-17 levels were substantially increased in the HIBD group compared with the control group. The temporal patterns of upregulation differed among the cytokines. The TNF- $\alpha$ mRNA expression increased at $3 \mathrm{~h}$ after HI injury compared with the basal level, and this level sharply differed from the control level at $24 \mathrm{~h}$. The IFN- $\gamma$ and IL-17 levels were significantly elevated at $24 \mathrm{~h}$, peaked at $3 \mathrm{~d}$, and then gradually decreased. Similar to the changes in the pro-inflammatory factors, the IL-10 levels in the brain also increased in the HIBD group, which increased at $6 \mathrm{~h}$ and peaked at $3 \mathrm{~d}$ (Figure $4 \mathrm{a}$ ). The expression TNF- $\alpha$, INF- $\gamma$, and IL-17 levels clearlydecreased in the PD-MSC group compared with the HIBD and fibroblast groups (all $P<0.01$ ). The IL-10 expression level was apparently greater in the three experimental groups; moreover, it notably increased in the PD-MSC group compared with the HIBD and fibroblast groups (all $P<0.01$; Figure $4 \mathrm{~b}$ ).

The soluble peripheral blood serum levels of IFN- $\gamma, \mathrm{TNF}-\alpha$, IL-17, and IL-10 were evaluated with enzyme-linked immunosorbent assays (ELISAs). The expression levels of pro-inflammatory cytokines were substantially upregulated after HI injury

a
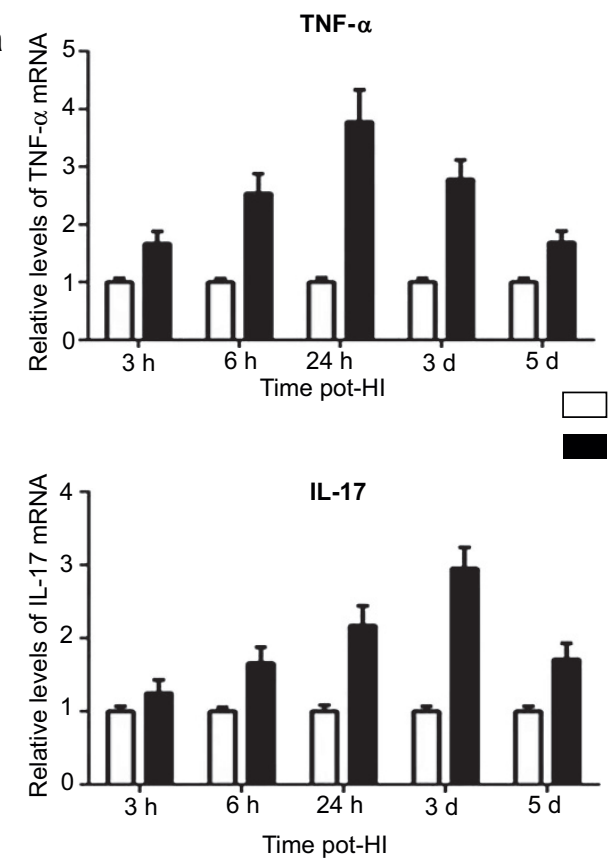

b

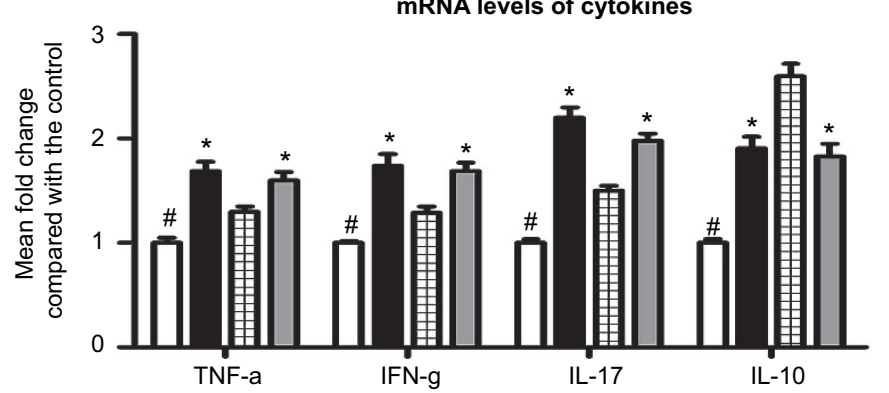
0.01 ; Figure $6 \mathrm{a}$ and $\mathrm{b}$ ). (control group: IFN- $\gamma: 10.83 \pm 2.13$; TNF- $\alpha: 22.40 \pm 4.35$; IL17: $4.92 \pm 1.08$. HIBD group: IFN- $\gamma: 43.56 \pm 7.12$; TNF- $\alpha$ : $67.93 \pm 8.11$; IL-17: $19.20 \pm 3.14$.). Compared with the fibroblast treatment, the PD-MSC treatment inhibited the production of pro-inflammatory cytokines and increased the production of IL-10 in the peripheral blood serum (PD-MSC group: IFN- $\gamma: 29.78 \pm 6.13$; TNF- $\alpha$ : $50.95 \pm 7.16$; IL-17: 12.06 \pm 3.21; IL-10: $41.84 \pm 6.17$. fibroblast group: IFN- $\gamma: 41.84 \pm$ 6.05; TNF- $\alpha$ : $64.49 \pm 8.31$; IL-17: $17.72 \pm 3.11$; IL-10: $26.58 \pm$ 4.25. all $P s<0.01$; Figure 5).

\section{PD-MSC treatment regulates Tregs after HIBD injury}

The flow cytometry results demonstrated that the number of Tregs was higher in the HIBD group $(10.43 \pm 1.29 \%)$ compared with the control group $(8.32 \pm 0.48 \%)$. The number of Tregs was greater in the PD-MSC group $(17.69 \pm 1.18 \%)$ compared with the fibroblast group $(13.14 \pm 1.06 \%, P<$

Western blot analysis demonstrated that the Foxp3 expression levels were clearly higher in the three experimental
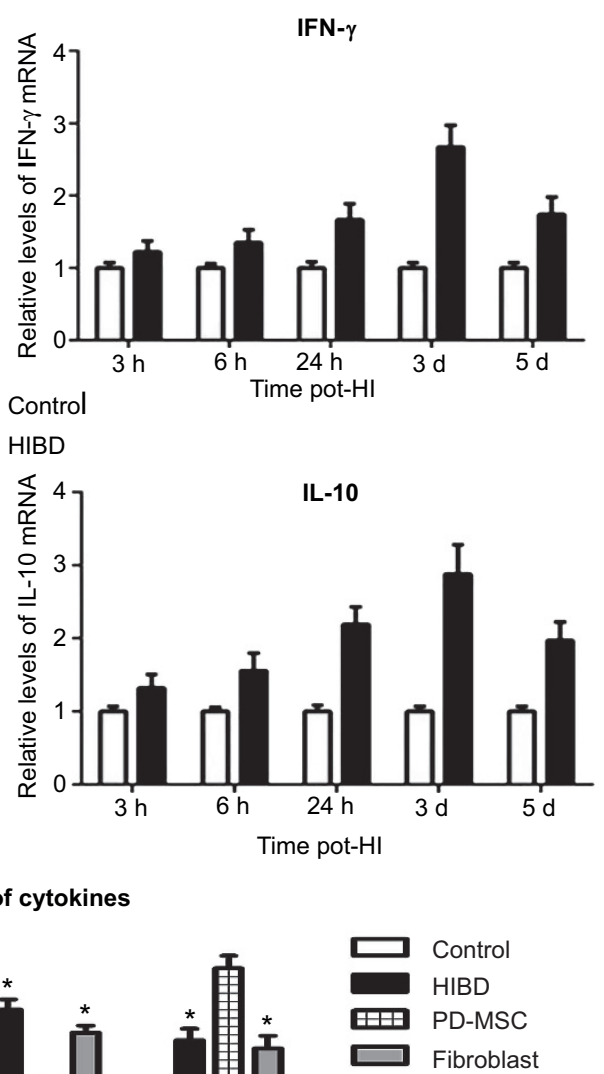

Figure 4 PD-MSC treatment attenuates systemic inflammation after $\mathrm{HI}$ injury at the gene transcription level. (a) An illustration of the dynamic TNF- $\alpha$, INF- $\gamma$, IL-17, and IL-10 mRNA expression levels. (b) The TNF- $\alpha$, INF- $\gamma$, IL-17, and IL-10 mRNA expressions levels 3 d after treatment. \#: $P<0.01$ vs. the control group; *: $P<0.01$ vs. the HIBD and fibroblast groups. 


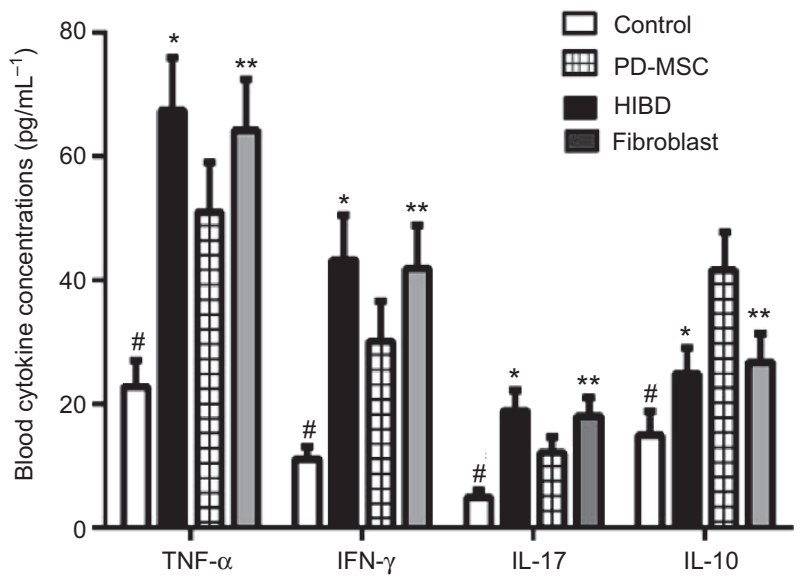

Figure 5 TNF- $\alpha$, IL-17, IFN- $\gamma$, and IL-10 blood cytokine concentrations for each groups. The IFN- $\gamma$, TNF- $\alpha$, and IL-17 expression levels were significantly higher in the HIBD group compared with the control group, whereas the expression levels in the PD-MSC group were lower than those in the HIBD group. The IL-10 levels were more significantly enhanced in the PD-MSC group than in the HIBD group. \#: $P<0.01$ vs. the control group; *: $P<0.01$ vs. the HIBD group; **: $P<0.01$ vs. the fibroblast group.

groups than in the control group (all $P<0.01$ ). In agreement with the flow cytometry results, the Foxp3 expression levels in the PD-MSC group were distinctly increased compared with those in the HIBD and fibroblast group (all $P<0.01$; Figure 6c).

\section{DISCUSSION}

Research has shown that MSC therapy has protective effects against $\mathrm{HI}$ injury. In our study, we found that a PD-MSC treatment significantly ameliorated movement disorders and increased the body weights of rats after HI injury. Moreover, the PD-MSC treatment reduced the infarct volume by suppressing the inflammatory cytokine upregulation in the damaged brain and induced Foxp3 expression.

We succeeded in isolating and culturing MSCs from the placenta. The adherent cells that were generated from the placenta tissues were plastic adherent, bore stromal surface markers (e.g. CD29, CD44, CD90, and CD105), and lacked hematopoietic cell markers (i.e. CD45). Moreover, these cells have adipogenic, cartilage, and osteogenic differentiation potentials. ${ }^{17}$ Thus, the placenta can be used as a source for fundamental research and cell treatment.

Over the past 10 years, several laboratories have studied MSCs in tissue repair models. ${ }^{18-20}$ Koh et al. ${ }^{21}$ have reported that 3 weeks after implantation, most hUC-MSCs are present in the injured hemisphere, and nestin expression in the hippocampus is increased. In our study, 6 hours after transplantation, GFP-positive PD-MSCs were mainly found at the injection site; however, $6 \mathrm{~d}$ after transplantation, the green fluorescent signal was significantly weakened. PD-MSCs mainly focus in the needle tract and injection site. The mechanisms of cell transplantation in brain injury treatment was previously considered to be the result of alternative nerve cells. However, now it is considered to be the result of a multifaceted a
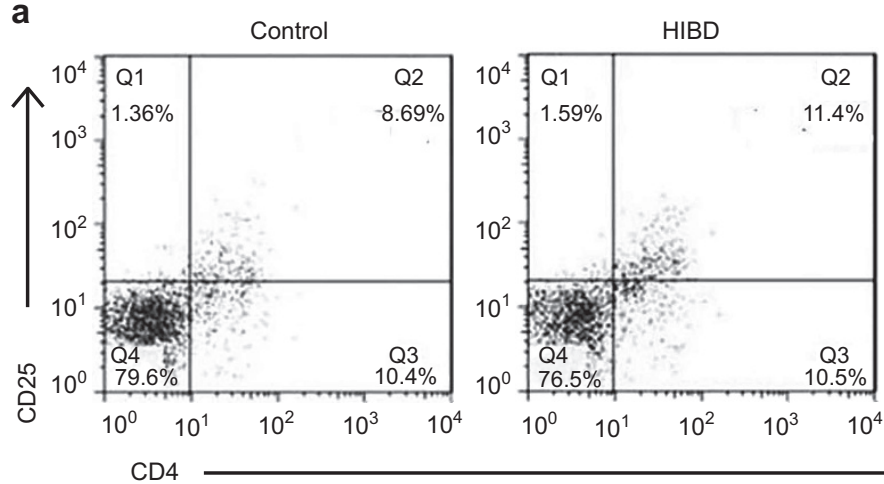

$10.5 \%$
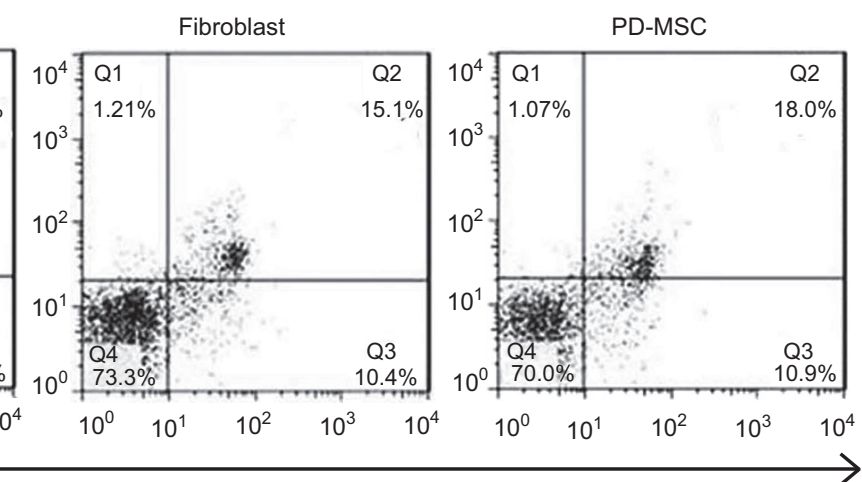

C

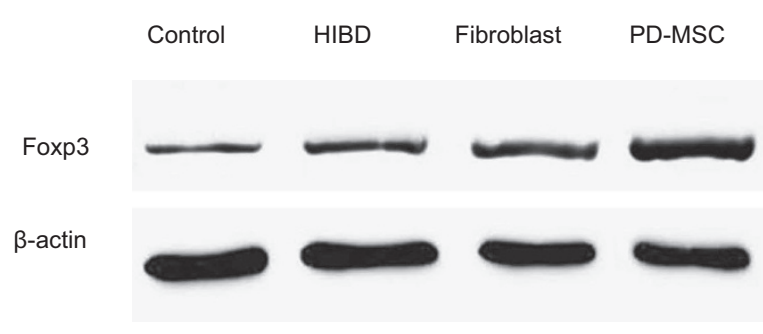

b

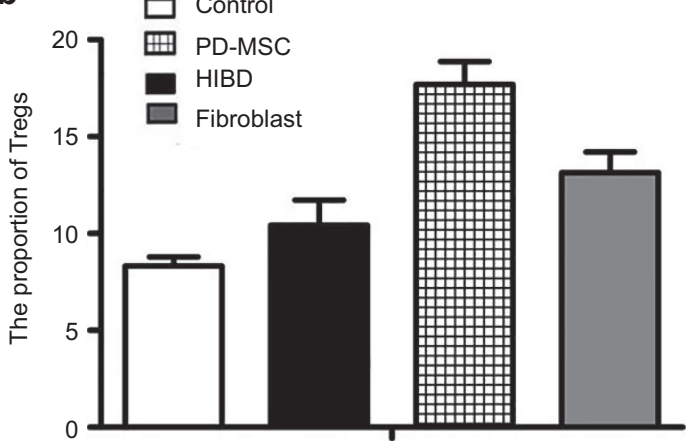

Figure 6 PD-MSC treatment regulates Tregs after HIBD injury. ( $\mathbf{a}$ and $\mathbf{b}$ ) Analysis of the number of Tregs by fluorescence-activated cell sorting. (c) Western blot analysis of the Foxp3 expression levels on $7 \mathrm{~d}$ after $\mathrm{HI}$ injury. 
joint comprehensive repair mechanism. The transplanted cells can not only directly replace damaged cells but also can promote endogenous neural stem cell repair, secretion of trophic factors, and local microcirculation improvements. ${ }^{22}$

Recently, some studies have focused on the role of cytokines in the evolution of HI injury. ${ }^{23}$ Following the destruction of the neuronal microenvironment and the blood-brain barrier, various immune cells migrate into the injured tissue and release inflammatory mediators. These inflammatory factors exaggerate cerebral edema and result in infarct expansion. At the early HI stages, macrophages, the main inflammatory effector cell, become visible early at $12 \mathrm{~h}$ and peak at $3 \mathrm{~d}$ after ischemiareperfusion. ${ }^{24}$ Macrophages promote postischemic inflammation, including the release of various inflammatory factors. TNF- $\alpha$ exerts neurotoxic effects mainly through the infiltration of leukocytes and the destruction of the blood-brain barrier. IFN- $\gamma$ directly leads to neuronal death. IL- 10 , an immunosuppressive cytokine, exerts neuroprotective effects after ischemic cerebral injury. Despite intensive studies, the mechanisms underlying brain inflammation remain unclear. ${ }^{25-27}$ Previous reports have failed to systematically study inflammatory cytokines and have used limited time points. The present study investigated the temporal expression levels of cytokines after $\mathrm{HI}$ injury from $3 \mathrm{~h}$ to $5 \mathrm{~d}$. The expression levels of pro-inflammatory cytokines markedly increased after HI brain injury. These data were in accordance with results from previous reports. These cytokines can infiltrate through the BBB, play a neuroprotective role in the pathophysiological process of HIBD, and aggravate cerebral edema and neurological damage. ${ }^{28}$ The notable changes in the cytokine expression levels in the hemispheres were time dependent. Thus, we hypothesized that these factors serve important functions in HIBD.

Following macrophage infiltration, $\mathrm{T}$ cells also migrate into the ischemic tissue and directly or indirectly cause neuronal cell death or dysfunction in the delayed process. After HI injury, Tregs have immunosuppressive effects that involve a variety of mechanisms, such as direct cell-cell action and the secretion of anti-inflammatory mediators. IL-10 plays a cerebroprotective role by suppressing the production of deleterious cerebral cytokines. ${ }^{5,29}$. In our research, the expression of IL-10 increased in the HIBD group. At $3 \mathrm{~d}$ after HI injury, the number of Tregs also clearly increased. These results indicate that Tregs participate in the inflammatory regulation in HIBD.

Our study corroborated that PD-MSC treatment can improve the development and functional recovery of rats after experimental HIBD. After cell transplantation, the rats in the PD-MSC group had no apparent brain atrophy, and their body weights even improved compared with the controls. Neurobehavioral tests showed that the PD-MSC group had the best recovery from HIBD injuse three treatment groups. However, the mechanisms by which MSCs enhance functional recovery are more complex than their multilineage differentiation potential and proliferative capacity. ${ }^{30,31}$ MSCs act via multifaceted pathways that remain incompletely identified.
Some recent studies have focused on the role of cytokines in the evolution of injury and have suggested that anti-inflammatory therapies reduce neurodegeneration and tissue damage after acute damage. ${ }^{32}$ In the current research, the TNF- $\alpha$, INF$\gamma$, and IL-17 expression levels were evidently lower than in the PD-MSC group. Additionally, IL-10 expression was markedly higher in the PD-MSC group. The number of Tregs and the Foxp3 expression level was also increased. Treatment with PDMSCs reduces the infarct volume by stimulating the generation of Tregs and suppressing the inflammatory reaction to brain tissue damage. ${ }^{33,34}$ From the present results, we infer that PDMSC treatment plays an immunomodulatory role by improving the balance between inflammatory and anti-inflammatory reactions in the pathogenesis of HIBD.

Overall, PD-MSC treatment can markedly restore brain tissue and improve functional outcomes. The key mechanisms underlying the neuroprotective effect of PD-MSC treatment on brain function may possibly involve the modulation of immune responses and the release of anti-inflammatory soluble factors.

\section{COMPETING INTEREST}

No conflict of interest.

\section{ACKNOWLEDGEMENTS}

The study was supported by grants from the Dongying City Technology Development Project (Grant No. 2011 106).

1 Lai MC, Yang SN. Perinatal hypoxic-ischemic encephalopathy. J Biomed Biotechnol 2011; 2011: 609813.

2 Scafidi J, Gallo V. New concepts in perinatal hypoxia ischemia encephalopathy. Curr Neurol Neurosci Rep 2008; 8: 130-138.

3 ladecola C, Anrather J. The immunology of stroke: from mechanisms to translation. Nat Med 2011; 17: 796-808.

4 Shichita T1, Sugiyama Y, Ooboshi H, Sugimori H, Nakagawa R, Takada I et al. Pivotal role of cerebral interleukin-17-producing gammadeltaT cells in the delayed phase of ischemic brain injury. Nat Med 2009; 15: 946-950.

5 Ryba M, Myśliwska J. CD4+CD25+Foxp3+ T lymphocytes: naturally occuring regulatory T cells. Pediatr Endocrinol Diabetes Metab 2010; 16: 289-294.

6 Liesz A, Suri-Payer E, Veltkamp C, Doerr H, Sommer C, Rivest S et al. Regulatory $\mathrm{T}$ cells are key cerebroprotective immunomodulators in acute experimental stroke. Nat Med 2009; 15: 192-199.

7 Gee JM, Kalil A, Shea C, Becker KJ. Lymphocytes: potential mediators of postischemic injury and neuroprotection. Stroke 2007; 38: 783788.

8 Dailey T, Mosley Y, Pabon M, Acosta S, Tajiri N, van Loveren $\mathrm{H}$ et al. Advancing critical care medicine with stem cell therapy and hypothermia for cerebral palsy. NeuroReport 2013; 24: 1067-1071.

9 Gonzales-Portillo GS, Reyes S, Aguirre D, Pabon MM, Borlongan CV. Stem cell therapy for neonatal hypoxic-ischemic encephalopathy. Front Neurol 2014; 5: 147.

10 Xia G, Hong X, Chen X, Lan F, Zhang G, Liao L et al. Intracerebral transplantation of mesenchymal stem cells derived from human umbilical cord blood alleviates hypoxic ischemic brain injury in rat neonates. J Perinat Med 2010; 38: 215-221.

11 Zhang W, Ou G, Hamrick M, Hill W, Borke J, Wenger K et al. Agerelated changes in the osteogenic differentiation potential of mouse bone marrow stromal cells. J Bone Miner Res 2008; 23: 1118-1128. 
12 Zhu SF, Zhong ZN, Fu XF, Peng DX, Lu GH, Li WH et al. Comparison of cell proliferation, apoptosis, cellular morphology and ultrastructure between human umbilical cord and placenta-derived mesenchymal stem cells. Neurosci Lett 2013; 541: 77-82.

13 Yasuhara T, Hara K, Maki M, Xu L, Yu G, Ali MM et al. Mannitol facilitates neurotrophic factor upregulation and behavioral recovery in neonatal hypoxic-ischemic rats with human umbilical cord blood grafts. J Cell Mol Med 2010; 14: 914-921.

14 Ding HF, Zhang H, Ding HF, Li D, Yi XH, Gao XY et al. Therapeutic effect of placenta-derived mesenchymal stem cells on hypoxicischemic brain damage in rats. World J Pediatr 2015; 11: 74-82.

15 Rice JE 3rd, Vannucci RC, Brierley JB. The influence of immaturity on hypoxic-ischemic brain damage in the rat. Ann Neurol 1981; 9: 131141.

16 Jacqueline N. Crawley. What's Wrong With My Mouse? Behavioral Phenotyping of Transgenic and Knockout Mice. John Wiley \& Sons, Inc, Hoboken, 2007; 72-73.

17 Nazarov I, Lee JW, Soupene E, Etemad S, Knapik D, Green W et al. Multipotent stromal stem cells from human placenta demonstrate high therapeutic potential. Stem Cells Trans/ Med 2012; 1: 359372.

18 Kawabori M, Kuroda S, Ito M, Shichinohe H, Houkin K, Kuge Y et al. Timing and cell dose determine therapeutic effects of bone marrow stromal cell transplantation in rat model of cerebral infarct. Neuropathology 2012; 33: 140-148.

19 Borlongan CV, Glover LE, Tajiri N, Kaneko Y, Freeman TB. The great migration of bone marrow-derived stem cells toward the ischemic brain: therapeutic implications for stroke and other neurological disorders. Prog Neurobiol 2011; 95: 213-228.

20 Zheng T, Rossignol C, Leibovici A, Anderson KJ, Steindler DA, Weiss MD. Transplantation of multipotent astrocytic stem cells into a rat model of neonatal hypoxic-ischemic encephalopathy. Brain Res 2006; 1112: 99-105.

21 Koh SH, Kim KS, Choi MR, Jung KH, Park KS, Chai YG et al. Implantation of human umbilical cord-derived mesenchymal stem cells as a neuroprotective therapy for ischemic stroke in rats. Brain Res 2008; 1229: 233-248.

22 Wu L, Leijten JC, Georgi N, Post JN, van Blitterswijk CA, Karperien M. Trophic effects of mesenchymal stem cells increase chondrocyte proliferation and matrix formation. Tissue Eng Part A 2011; 17: 1425-1436.

23 Wang $Q$, Tang $X N$, Yenari MA. The inflammatory response in stroke. J Neuroimmunol 2007; 184: 53-68.
24 Clausen BH, Lambertsen KL, Babcock AA, Holm TH, DagnaesHansen F, Finsen B. Interleukin-1 beta and tumor necrosis factoralpha are expressed by different subsets of microglia and macrophages after ischemic stroke in mice. J Neuroinflammation 2008; 5: 46.

25 Xiong X, Barreto GE, Xu L, Ouyang YB, Xie X, Giffard RG. Increased brain injury and worsened neurological outcome in interleukin-4 knockout mice after transient focal cerebral ischemia. Stroke 2011; 42: 2026-2032.

26 Zhu Y, Yang GY, Ahlemeyer B, Pang L, Che XM, Culmsee C et al. Transforming growth factor-beta 1 increases bad phosphorylation and protects neurons against damage. J Neurosci 2002; 22: 38983909.

27 Sun Y, Calvert JW, Zhang JH. Neonatal hypoxia/ischemia is associated with decreased inflammatory mediators after erythropoietin administration. Stroke 2005; 36: 1672-1678.

28 Shichita T, Ago T, Kamouchi M, Wu F, Zhang W, Ooboshi H. Nove therapeutic strategies targeting innate immune responses and early inflammation after stroke. J Neurochem 2012; 123: 29-38.

29 Liu WS, Chen CT, Foo NH, Huang HR, Wang JJ, Chen SH et al. Human umbilical cord blood cells protect against hypothalamic apoptosis and systemic inflammation response during heatstroke in rats. Pediatr Neonatol 2009; 50: 208-216.

30 Fan CG, Zhang QJ, Tang FW, Han ZB, Wang GS, Han ZC. Human umbilical cord blood cells express neurotrophic factors. Neurosci Lett 2005; 380: 322-325.

31 Newman MB, Willing AE, Manresa JJ, Sanberg CD, Sanberg PR. Cytokines produced by cultured human umbilical cord blood (HUCB) cells: implications for brain repair. Exp Neurol 2009; 199 : 201-208.

32 Jenkins DD, Rollins LG, Perkel JK, Wagner CL, Katikaneni LP, Bass WT et al. Serum cytokines in a clinical trial of hypothermia for neonatal hypoxic-ischemic encephalopathy. J Cereb Blood Flow Metab 2012; 32: 1888-1896.

33 Ghannam S, Pène J, Torcy-Moquet G, Jorgensen C, Yssel H. Mesenchymal stem cells inhibit human Th17 cell differentiation and function and induce a $\mathrm{T}$ regulatory cell phenotype. $J$ Immunol 2010; 185: 302-312.

34 Rafei M, Campeau PM, Aguilar-Mahecha A, Buchanan M, Williams P, Birman E et al. Mesenchymal stromal cells ameliorate experimental autoimmune encephalomyelitis by inhibiting CD4 Th17 T cells in a CC chemokine ligand 2-dependent manner. J Immunol 2009; 182 5994-6002. 